\title{
Performance of a Large Area Mercuric Iodide Lung Counter
}

\author{
M. W. Mallett, M. E. Ennis \\ Los Alamos National Laboratory \\ Health Physics Measurements, HSR-4 \\ MS G761, Los Alamos, NM 87545 USA \\ mallett@lanl.gov
}

\author{
A. E. Proctor, L. van den Berg, J. W. Hintenach \\ Constellation Technology Corporation \\ 7887 Bryan Dairy Road, Suite 100 \\ Largo, FL 33777 USA \\ lvdberg@contech.com
}

\begin{abstract}
A new detector system based on mercuric iodide technology was designed and constructed specifically for in vivo measurement applications. The system contains four mercuric iodide room-temperature semiconductor detectors, each with an area of $25 \mathrm{~mm} \times 25 \mathrm{~mm}$, for photon detection and spectrometry. The combination of multiple matched detectors in a single housing achieves the large area needed for in vivo measurements, while overcoming the inherent limitations in the growth of large, high quality crystals of high-Z semiconductor materials. The use of large semiconductor detectors operating at room temperature eliminates the need for liquid nitrogen cooling required for germanium detectors which presents a potential danger in the confined space characteristic of in vivo measurements. The high quality room temperature detectors preserve the spectroscopic quality requirements for transuranic detection. The performance of the mercuric iodide in vivo detector meets the performance requirements specified in ANSI N13.30, and compares satisfactorily with the performance of germanium detector systems typically used for in vivo lung measurements.
\end{abstract}

Keywords-mercuric iodide; array; radiobioassay; in vivo; lung.

\section{INTRODUCTION}

Historically, the measurement of low-energy photons required deciding between two competing spectroscopy qualities-improved detection efficiency provided by scintillators $(\mathrm{NaI})$, or improved energy resolution afforded by semiconductors (HPGe). In an attempt to achieve both qualities for in vivo measurements, systems featuring multiple germanium detectors have been employed to increase efficiency via increased surface area. The Los Alamos National Laboratory's (LANL) In Vivo Measurement Laboratory (IVML) utilizes such a system incorporating six HPGe detectors. The primary operational drawback to this scenario is the requirement of a large amount of liquid nitrogen cooling in a confined space (inside the in vivo shielding). The large liquid nitrogen dewars needed to cryogenically cool the germanium crystals also yielded a system too cumbersome to routinely position each detector independently for a given subject.

Investigations of other semiconductor materials with higher effective atomic number $(Z)$ have been made in an effort to improve detection efficiency while maintaining superb energy resolution [1-2]. Mercuric iodide $\left(\mathrm{HgI}_{2}\right), Z=80-53$, is one

A.E. Proctor's current address is Nucsafe, Inc., 765 Emory Valley Road, Oak Ridge, TN 37830.

This research was sponsored by the HSR-Division Technology Development, Evaluation, and Application Program, LANL, Los Alamos, such material. One benefit of this technology is that due to the large bandgap width $(2.1 \mathrm{eV})$, a detector based on $\mathrm{HgI}_{2}$ can be operated at room temperature. Thus, the need for liquid nitrogen reservoirs is eliminated. However, the principal obstacle to an in vivo system based upon $\mathrm{HgI}_{2}$ has been the inability to manufacture crystals of sufficient size to yield a practical detector. For example, $\mathrm{HgI}_{2}$ crystals are now commercially available up to $25 \mathrm{~mm} \times 25 \mathrm{~mm}$ and $1-2 \mathrm{~mm}$ in thickness. This compares unfavorably to commonly used HPGe detectors having active areas of $2000-4000 \mathrm{~mm}^{2}$.

One solution would be to electrically couple multiple $\mathrm{HgI}_{2}$ crystals in a single detector housing. Four $25 \mathrm{~mm}$ x $25 \mathrm{~mm}$ crystals could be arrayed to form a single large area detector identical in size to a single $2500 \mathrm{~mm}^{2}$ HPGe detector. The circuitry should accommodate a common bias, separate detector preamps, and separate signal outputs for each of the detectors in the array.

\section{DETECTOR DESIGN}

Constellation Technology (CT) and LANL designed, constructed, and performed the initial characterization of a large area mercuric iodide detector suitable for in vivo measurement application. The circuit board design is shown in Fig. 1. The mercuric iodide lung counter is shown in Fig. 2.

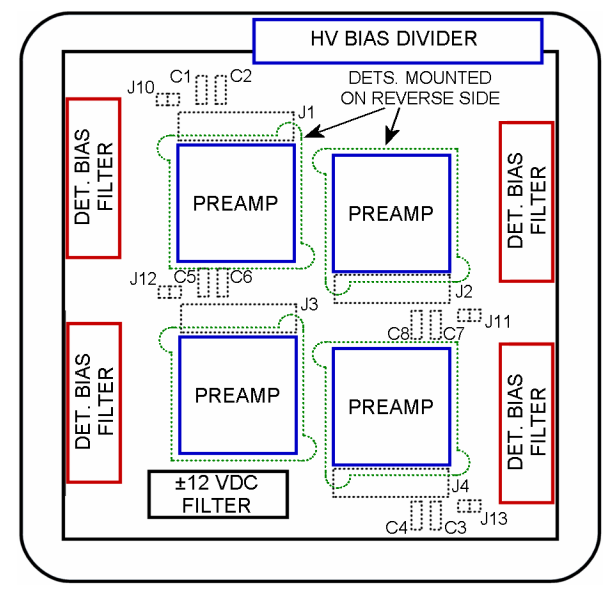

Figure 1. Circuit board and component location design, rear side. 


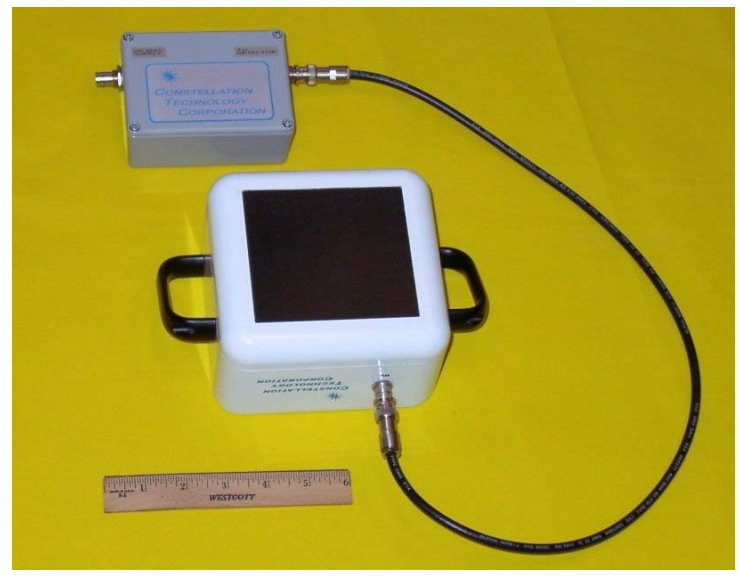

Figure 2. Mercuric iodide array lung counter.

The lung counter features four $25 \mathrm{~mm} \times 25 \mathrm{~mm} \mathrm{HgI}_{2}$ crystals. The total active area is $2500 \mathrm{~mm}^{2}$. Each crystal is approximately $1.5 \mathrm{~mm}$-thick. One high voltage (HV) input and four separate signal output connections are present on the detector housing. Gain matching and spectral summing is achieved via external shaping amplifiers and MCA software.

The NIM bias supply is nominally set at $+1500 \mathrm{~V}$. A HV divider is used so that a single supply can bias each of the four detectors. The divider outputs are fed to separate RC-filters for each detector. Individual detector bias levels were selected to yield the best resolution. Detector performance was optimized for transuranic photon emissions, $\sim 10-200 \mathrm{keV}$.

An in-line backup battery supply containing dry cell batteries maintains bias during power failure. The $+135 \mathrm{~V}$ bias permits rapid recovery, allowing the full operating voltage to be reapplied instantaneously. Internal diodes prevent current flow through the batteries when the detector is biased using the NIM supply.

A separate preamp is provided for each detector. The eV Products ${ }^{1}$ Model eV-5093 preamp is an ultra-low noise, charge-sensitive unit specifically intended for use with $\mathrm{HgI}_{2}$ detectors. The onboard $12 \mathrm{VDC}$ filter supplies power to the preamps. The capacitance of the detectors in the lung counter has been measured as $30-35 \mathrm{pF}$. This corresponds to an approximate $2.5 \mathrm{keV}$ FWHM noise level from the preamp.

The end window of the lung counter housing is $0.76-\mathrm{mm}$ thick carbon fiber-epoxy. It has been coated with paint to minimize light entry inside the unit. Also, the inner window surface is coated with a thin (100's of angstroms) metal EMI shield. The thin window exhibits effectively $100 \%$ photon transmission above $40 \mathrm{keV}$.

\section{CHARACTERIZATION METHODS}

Characterization of the $\mathrm{HgI}_{2}$ lung counter was performed at LANL following the tenets of ANSI N13.30, Performance Criteria for Radiobioassay [3]. This standard prescribes the method for performing in vivo calibrations as well as acceptable detection limits to warrant accreditation. Lung measurement calibrations were performed using the standard torso phantom as shown in Fig. 3.

\footnotetext{
'eV Products, 373 Saxonburg Blvd., Saxonburg, PA 16056.
}
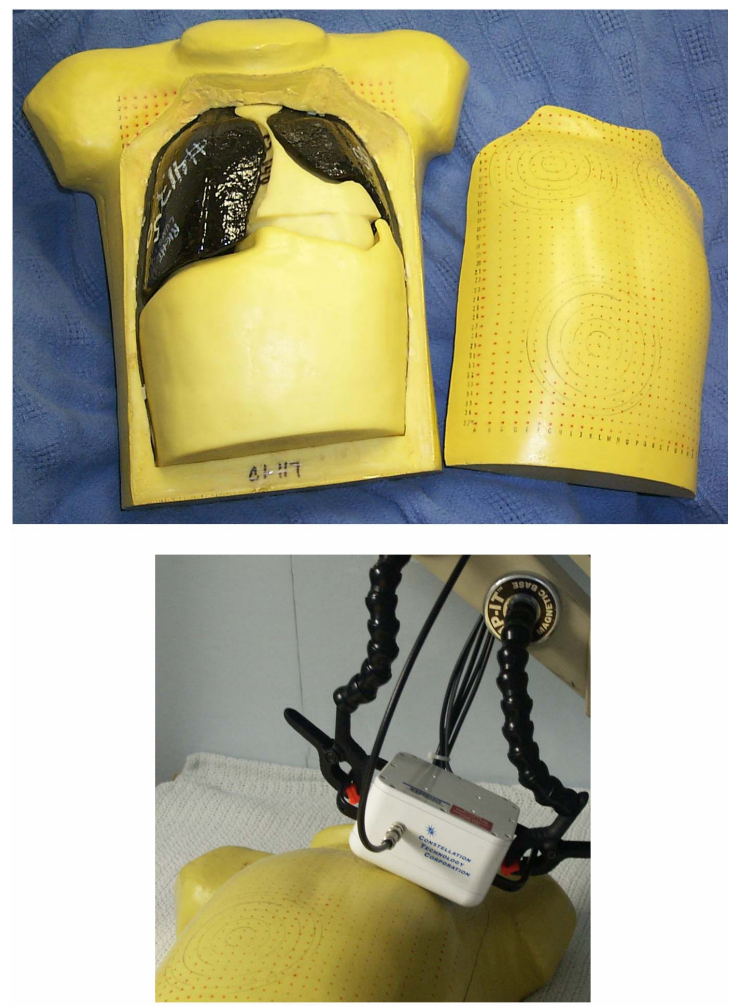

Figure 3. Torso phantom calibration. Lung sets have uniformly distributed radioactivity (top). The lung counter is suspended from a dental arm using flexible arm clamps (bottom).

The torso phantom is constructed of tissue equivalent materials including a rib cage in the anterior base chest plate. Lung sets are available for different radionuclides (e.g. Am241, Pu-238/-239, Eu-152, fission products, etc.) distributed uniformly throughout the lung/air-equivalent material. The base chest plate has a mean chest wall thickness of $1.63 \mathrm{~cm}$.

The performance criteria of ANSI N13.30 defines the minimum detectable amount (MDA) in simplified form as

$$
\mathrm{MDA}=\frac{\left(4.65 s_{b}+3\right)}{K T},
$$

where $s_{b}$ is the standard deviation of a total blank count (e.g. a non-contaminated person) and $K$ is the calibration factor at the peak energy (counts/photon). At LANL, the standard subject counting time, $T$, is $2000 \mathrm{~s}$. Inherent in the definition is a $5 \%$ probability of Type I and Type II errors $(\alpha=\beta=0.05)$.

\section{RESUlTS AND CONCLUSIONS}

Characterization testing of the $\mathrm{HgI}_{2}$ lung counter was performed using an Ametek ORTEC ${ }^{2}$ Model 572A shaping amplifier with a selected $10 \mu$ s shaping time. Data collection was made using a MicroACE ${ }^{\mathrm{TM}}$ MCA card with a selected ADC conversion gain of 2048 channels. An Am-241 spectrum collected for one of the four array detectors is shown in Fig. 4. The 26, 33 and $59.5 \mathrm{keV}$ photon lines are clearly distinguishable. However, the 14, 17, and $20 \mathrm{keV}$ lines cannot be differentiated.

\footnotetext{
${ }^{2}$ Ametek ORTEC, 801 South Illinois Avenue, Oak Ridge, TN 37830.
} 


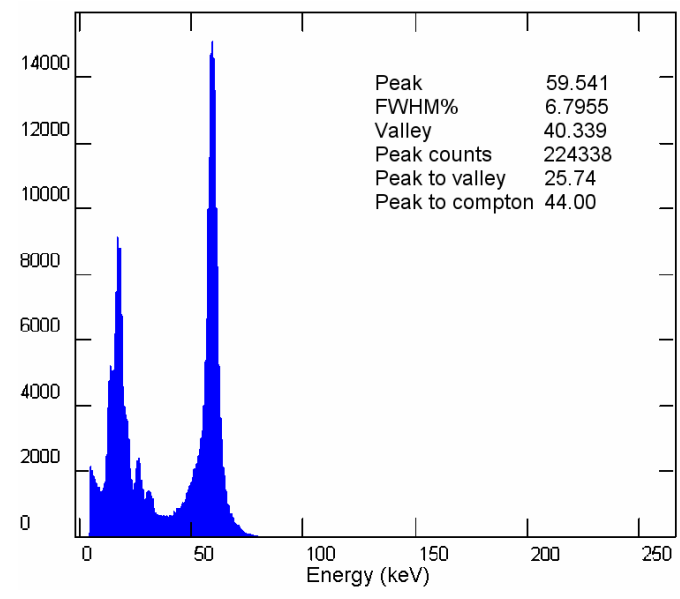

Figure 4. Lung counter spectrum for Am-241.

The measured energy resolution of the $\mathrm{HgI}_{2}$ lung counter was $6.2 \mathrm{keV}(5.0 \%)$ at $122 \mathrm{keV}$. Additional results are given in Table I for the array. Also reported is the measured resolution of the Princeton Gamma-Tech ${ }^{3}$ Model NIGP 2020 HPGe planar detector currently used in the IVML six-detector system.

Calibration of the $\mathrm{HgI}_{2}$ lung counter was made using the torso phantom. Measurements were made using several lung sets, including Am-241, enriched uranium (U-235), and Ba133. All measurements were performed using the base chest plate with the lung counter anterior to the right lung. From this data, the calibration factor, $K$, was determined over the useful energy range of the detector $(5-200 \mathrm{keV})$.

TABLE I. $\quad \mathrm{HGI}_{2}$ LUNG COUNTER ENERGY RESOLUTION

\begin{tabular}{|c|c|c|}
\hline \multirow{2}{*}{$\begin{array}{c}\text { Energy } \\
(\mathrm{keV})\end{array}$} & \multicolumn{2}{|c|}{ FWHM (keV) } \\
\cline { 2 - 3 } & HgI $_{2}$ Lung Counter & IVML HPGe Detector \\
\hline 14.4 & 2.3 & 0.66 \\
\hline 59.5 & 4.0 & 0.70 \\
\hline 122 & 6.2 & 0.85 \\
\hline
\end{tabular}

MDA results for the $\mathrm{HgI}_{2}$ lung counter are given in Table II. All results are based on a $2000 \mathrm{~s}$ count time, T. Also reported are MDA results for the current IVML six-HPGe detector system. Note that the results cited for the $\mathrm{HgI}_{2}$ system are for a single lung counter (four detectors with $2500 \mathrm{~mm}^{2}$ total active area) whereas the IVML results are for a system featuring six HPGe detectors $\left(12,000 \mathrm{~mm}^{2}\right.$ total active area).

The results of Table II show that the $\mathrm{HgI}_{2}$ lung counter satisfies the performance requirements of ANSI N13.30. The lung counter is approximately $25 \%$ more efficient than a single HPGe detector. This is equivalent to the increase in active area of the $\mathrm{HgI}_{2}$ array $\left(2500 \mathrm{~mm}^{2}\right.$ vs. $2000 \mathrm{~mm}^{2}$ ). Approximately 4.7 $\mathrm{HgI}_{2}$ lung counters would have the same detection efficiency as the IVML six-HPGe detector system.

The $\mathrm{HgI}_{2}$ lung counter also represents a mobile system by virtue of the in-line backup battery. Negligible discharge of the crystal bias was observed following more than six months with no external bias applied. The full operating voltage was reapplied instantaneously. As such, the system is suitable for emergency response applications where portability is required.

TABLE II. $\quad \mathrm{HGI}_{2}$ LUNG COUNTER MDA

\begin{tabular}{|c|c|c|c|}
\hline \multirow{2}{*}{ Radionuclide } & \multicolumn{3}{|c|}{ Minimum Detectable Amount, MDA (Bq) } \\
\cline { 2 - 4 } & $\begin{array}{c}\text { Minimum Test } \\
\text { Level }\end{array}$ & $\begin{array}{c}\text { HgI }_{2} \text { Lung } \\
\text { Counter }\end{array}$ & $\begin{array}{c}\text { IVML HPGe } \\
\text { Detector }^{\boldsymbol{a}}\end{array}$ \\
\hline Am-241 & 100 & 38 & 8 \\
\hline Th-234 & 500 & 340 & 75 \\
\hline U-235 & 30 & 28 & 5 \\
\hline
\end{tabular}

\section{REFERENCES}

[1] "Mercuric iodide nuclear radiation detectors, proceedings of the fifth international workshop on mercuric iodide nuclear radiation detectors," in Nuc. Instr. Meth. Phys. Res., vol. 213, no. 1, M. M. Schieber and L. van den Berg, Eds. Amsterdam: North-Holland Publishing Company, 1983, pp. 1-154.

[2] J. S. Iwanczyk and A. J. Dabrowski, "Large area mercuric iodide photodetectors," in IEEE Trans. Nucl. Sci., vol. NS-31, no. 1, pp. 336339, February 1984

[3] American National Standards Institute, Inc., An American National Standard - Performance Criteria for Radiobioassay, HPS N13.30-1996. McLean, VA: Health Physics Society, 1996.
${ }^{3} \mathrm{~N}$-type detector, $50 \mathrm{~mm}$ in diameter, $20 \mathrm{~mm}$-thick, $0.51 \mathrm{~mm}$-thick beryllium window, $-2500 \mathrm{~V}$ bias. Princeton Gamma-Tech, 1026 Route 518, Rocky Hill, NJ 08553. 